\title{
The Validation of Exact Mass Measurements for Small Molecules Using FT-ICRMS for Improved Confidence in the Selection of Elemental Formulas
}

\author{
Julie M. Herniman and G. John Langley \\ University of Southampton, School of Chemistry, Southampton, United Kingdom
}

\author{
Tony W. T. Bristow and Gavin O'Connor \\ LGC Limited, Teddington, Middlesex, United Kingdom
}

The confidence in an individual measurement is the most important factor when selecting the elemental formula candidates from the list of possible elemental compositions following an exact mass measurement. It is the single mass measurement capability rather than the averaged mass measurement potential of the mass spectrometer that is the critical factor when validating the exact mass measurements of small molecules. Here, an experimental protocol has been established to determine the frequency of exact mass measurement by Fourier transform ion cyclotron resonance mass spectrometry (FT-ICRMS) at known relative ion abundance ratios (RA). This in turn allows for statements about the confidence limit for any single exact mass measurement to be made. This is particularly crucial for a high throughput, automated environment where operator intervention is required to be minimal and repeat analyses are to be avoided. The relative ion abundance calculations are essential to determine the working ranges for specific sample ion abundances. Further, it has been shown that if the sample ion abundance is low, then the ion abundance range for the calibration file does not need to be exactly or closely matched, again benefiting the high throughput application. (J Am Soc Mass Spectrom 2005, 16, 1100-1108) (c) 2005 American Society for Mass Spectrometry

$\mathrm{E}$ xact mass measurement is used to identify the elemental formula of specific ions. Historically, this has been a manual and labor intensive approach via magnetic sector instrumentation [1]. With the advent of further development of new modern instrument types, in particular FT-ICRMS and time-offlight mass spectrometry (TOFMS), exact mass measurement has become a more accessible technique. The data is used to uniquely identify molecules from many different areas, e.g., novel synthetic compounds, ratification of known synthetic pathways, drug discovery, drug metabolism, pharmacognosy, and many more.

For publication purposes, learned journals accept either elemental analysis or exact mass measurement for identification of novel compounds. The demand for quick, exact mass measurements has increased rapidly over recent years because of the constraints of elemental analysis, sample size and composition, and the increasing number of samples now produced following the

Published online May 25, 2005

Address reprint requests to Dr. G. J. Langley, University of Southampton, School of Chemistry, Highfield, Southampton SO17 1BJ, UK. E-mail: gil@soton.ac.uk combinatorial chemistry revolution. In the case of an unknown, there needs to be a high level of confidence in the measurement obtained, together with knowledge of the accuracy, precision and uncertainty of the selected method. Selection of the appropriate elemental formula(s) must be based on the performance of the instrument and not on arbitrary limits that may be set by journals, e.g., Journal of Organic Chemistry states "For most new compounds, HRMS data accurate within 5 ppm or combustion elemental analysis data accurate within $0.4 \%$ should be reported to support the molecular formula assignment" [2], or "cherry picked" by the user.

In a high throughput, open access mass spectrometry environment fast, reliable exact mass measurement instrumentation is essential to relieve any analytical bottlenecks. FT-ICRMS has been shown to offer the highest mass resolving power, resolution, and accuracy of any mass spectrometer [3], and has been routinely used for exact mass measurement with mass accuracy of less than or equal to $1 \mathrm{ppm}$ [4]. Zhang et al. extensively reviewed the intrinsic worth of FT-ICRMS and associated space charge issues [5], whilst a recent round-robin study has shown that mass accuracy better 


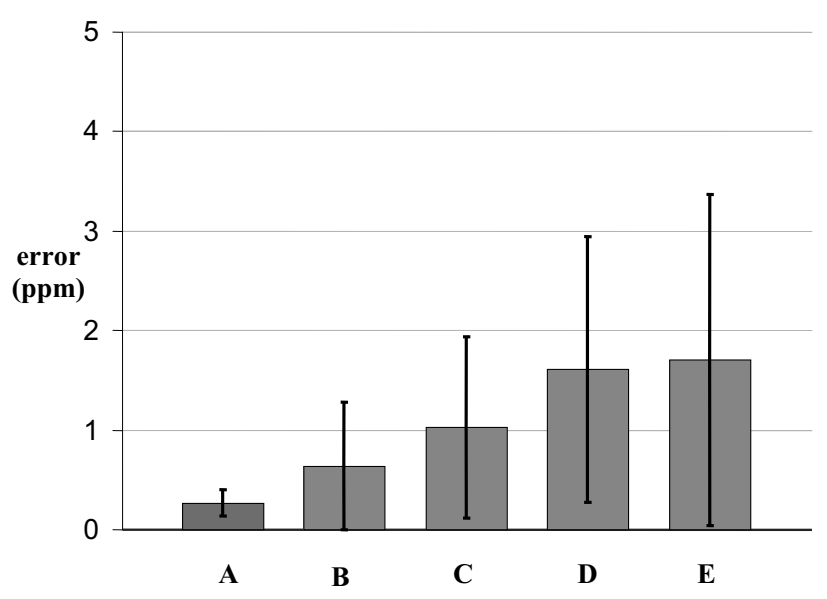

Figure 1. Typical FT-ICRMS exact mass data from Laboratory A showing average error and standard deviations obtained using various methods of analysis and data handling. A-Internally calibrated infusion data $(\mathrm{n}=20)$; $\mathbf{B}$-externally calibrated infusion data $(\mathrm{n}=100)$; $\mathbf{C}$-externally calibrated automated FIA data, verified by mass spectrometrist $(n=300)$; $\mathbf{D}$-externally calibrated automated FIA data, high and low intensity data discounted $(\mathrm{n}=$ 250); E-externally calibrated automated FIA data, all data, no criteria applied $(\mathrm{n}=800)$.

than $1 \mathrm{ppm}$ can readily be achieved using FT-ICRMS with the implementation of certain external calibration protocols [6]. Further, ESI-FT-ICRMS exact mass measurement has been successfully automated in the pharmaceutical industry, where the accuracy and precision of the instrument has been essential to assigning formulas for expected compounds [7]. Here, an arbitrary limit of $5 \mathrm{ppm}$ has been stipulated, if the sample measurement falls below this mass error then the measurement is accepted and the results returned to the chemist. The robust nature of this approach has been proven since errors greater than $5 \mathrm{ppm}$ for the correct formulas are not often encountered [7].

The space charge effects described by Taylor and Amster [8] and approaches to address this, as discussed by Zhang and coworkers [5], are particularly relevant where there is very little or no control over sample concentration or purity. Improvements could be achieved using internal calibration, thus correcting for any frequency shift (hence $\mathrm{m} / \mathrm{z}$ ) experienced by the ions in the FT-ICRMS cell. However, this approach is not necessarily desirable for a high throughput environment.

Figure 1 shows the typical data for open access FTICRMS exact mass measurement from one of the laboratories in this study. For comparison, Range A shows the average error and standard deviation for internally calibrated exact mass measurements via infusion of sample using a syringe driver. Range B illustrates the externally calibrated data from infusion experiments where the mass spectrometrist makes on-the-fly decisions about matching ion populations, peak intensities, and other factors whereas Range $\mathrm{E}$ is the fully automated data acquisition and the results following automatic data processing. These data sets are in good agreement with the work of Dykes and coworkers [7].

The implementation of simple criteria to help address issues such as poor peak profile, large differences in ion populations, over abundant ion populations, and space charging gives rise to the improvements shown in Ranges D and C. Although good average errors are obtained at all stages and improvements in these shown at the different Ranges from E to B, the distribution of the measurements and standard deviations are initially large but also improve across the data set (E to B).

The aim of this work is to understand the major issues that cause the large standard deviations, to allow the implementation of an experimental protocol that addresses this, thus removing the need for post acquisition intervention by the analyst. This is essential for reliability and confidence in each individual measurement recorded. This would then diminish the need for intervention by the spectrometrist and move toward routinely working in Range B, thus leading to improved confidence in elemental formula selected by the instrumental software following an exact mass measurement. The importance of this is clearly demonstrated by the data in Table 1.

Table 1. Examples of the number of theoretical elemental formulae candidates for three different known species at nominal masses $m / z 200,500$ and 700 .

\begin{tabular}{|c|c|c|c|c|c|c|}
\hline \multirow[b]{2}{*}{ Title [Theoretical mass] } & \multirow{2}{*}{$\begin{array}{l}\text { Formula } \\
\text { Elements }\end{array}$} & \multicolumn{5}{|c|}{ Cumulative number of theoretical candidates } \\
\hline & & $0.1 \mathrm{ppm}$ & $1 \mathrm{ppm}$ & $2 \mathrm{ppm}$ & $3 \mathrm{ppm}$ & 5 ppm \\
\hline \multirow[t]{4}{*}{ Example 1 [200.10699] } & $\mathrm{C}_{13} \mathrm{H}_{14} \mathrm{NO}$ & & & & & \\
\hline & CHNO & 1 & 1 & 1 & 1 & 1 \\
\hline & CHNONa & 1 & 1 & 1 & 1 & 1 \\
\hline & CHNOSPIFSiNa & 1 & 3 & 7 & 12 & 24 \\
\hline \multirow[t]{4}{*}{ Example 2 [500.18161] } & $\mathrm{C}_{28} \mathrm{H}_{26} \mathrm{~N}_{3} \mathrm{O}_{6}$ & & & & & \\
\hline & CHNO & 2 & 2 & 8 & 12 & 18 \\
\hline & CHNONa & 2 & 3 & 15 & 21 & 35 \\
\hline & CHNOSPIFSiNa & 78 & $>500$ & & & \\
\hline \multirow[t]{4}{*}{ Example 3 [700.27121] } & $\mathrm{C}_{34} \mathrm{H}_{42} \mathrm{~N}_{3} \mathrm{O}_{13}$ & & & & & \\
\hline & CHNO & 3 & 6 & 12 & 20 & 28 \\
\hline & CHNONa & 3 & 12 & 30 & 41 & 67 \\
\hline & CHNOSPIFSiNa & 321 & $>500$ & & & \\
\hline
\end{tabular}


Table 1 shows the number of theoretical candidates for the three simple compounds listed. In Example 1, if the compound composition is known to be limited to $C$, $\mathrm{H}, \mathrm{O}$, and $\mathrm{N}$, then at the 5 ppm level there is only one candidate. If the compound is of unknown composition, more elements must be included in the calculation. For illustration in this example, simple monoisotopic elements are used, i.e., elements that have easily recognizable isotope patterns such as $\mathrm{Cl}$ or $\mathrm{Br}$ are omitted. For a compound of relative molecular mass 200, the number of candidates now dramatically increases from 1 to 24, see Example 1. As the mass of the analyte increases, the number of likely candidates dramatically grows, see Example 3, where the composition is limited to $\mathrm{C}, \mathrm{H}, \mathrm{O}$, and $\mathrm{N}$ then at the 5 ppm level there are 28 candidates; this increases to 67 candidates if $\mathrm{Na}$ is added to the list of elements. At this stage, additional chemical and mass spectrometric knowledge can be applied to the assignment, e.g., formulas that disobey the nitrogen rule or are odd electron species can be discounted and hence, the number of candidates reduced.

In Figure 1 column E, the average error in exact mass measurement is less than $2 \mathrm{ppm}$. The standard deviation is nearly double this but neither of these figures gives the confidence weighting of the individual measurement. It is the spread of measurements, coupled with the factors that contribute to the measurement variability, which can be used to limit the distribution of elemental formulas. It is these factors which are needed experimentally to set the confidence limit for each analysis. Once established, the experimentally determined error range can be used to determine the number of candidates. The average mass measurement capability of the instrument should not be used to determine this error range or give confidence to a single measurement.

This study set out to determine and understand the factors that influence the range of mass measurement errors recorded, then to apply this knowledge to improve the experimental protocol to define the confidence limit of a single mass measurement. This was accomplished by measuring the same compound at different sample concentrations, cross-calibrated with calibration solutions of equivalent concentrations. To minimize instrument specific characteristics the performance of two separate FT-ICRMS systems was compared.

The total ion abundances at each concentration, for sample and calibrant, were calculated along with the ion abundance ratio of calibrant:sample for each measurement. By comparing the two sets of data, from the separate instruments, a better understanding of the factors affecting instrument performance which give rise to the accuracy and precision of exact mass measurement in FT-ICRMS have been achieved. The level of confidence for the exact mass measurement of a small molecule analyzed, using FT-ICRMS with external calibration, was determined and an experimental protocol established and tested via a blind trial. This has led to greater and improved confidence in selection of elemental formulas candidates.

\section{Experimental}

Initial investigations involved the analysis of a single unknown compound $\left(\mathrm{m} / \mathrm{z} 376[\mathrm{M}+\mathrm{H}]^{+}\right)$at three different concentrations, $0.1,1.0$, and $10 \mu \mathrm{g} \mathrm{mL}{ }^{-1}$ in methanol. The sample at each concentration was analyzed five times via infusion on five separate days on the two Bruker FT-ICRMS systems. Each instrument was optimized and tuned to the operator's normal working conditions although both were calibrated using each of the four identical calibration mixtures at 0.1 , $1.0,10$, and $100 \mu \mathrm{g} \mathrm{mL}^{-1}$ per component.

Throughout this comparison, the instruments were used in a manner to mimic a high throughput environment, i.e., operator intervention was kept to a minimum with source and instrument cleaning minimal.

Exact mass measurement was then performed on the sample at each of the three sample concentrations. Four separate external calibration files were created from each one of the three calibration solutions. All sample spectra were then calibrated with each one of the four external calibration files.

This gives a total of 60 measurements for each instrument from each day of the study, however, for Laboratory B the calibration solutions at $100 \mu \mathrm{g} \mathrm{mL} \mathrm{m}^{-1}$ per component were rejected because of peak splitting issues. The total ion abundance for each sample and calibration spectrum was then calculated and the ppm error for each measurement was recorded. The relative ion abundance ratio for each calibrant concentration to each sample concentration was then determined. The data obtained from both laboratories was compared and some preliminary protocols established.

\section{Fourier Transform-Ion Cyclotron Resonance Mass Spectrometry}

All experiments were carried out using a Bruker Daltonics (Billerica, MA) Apex III FT-ICRMS system in Laboratories A and B, both equipped with a 4.7 Tesla actively shielded superconducting magnet and an Infinity cylindrical analyzer cell. The samples were introduced to the mass spectrometer using an Apollo off-axis electrospray ionization source. The capillary voltage was set at $-4500 \mathrm{~V}$. The drying gas flow rate of 30 arbitrary units and nebulizing gas pressure of 50 psi were applied to the nebulizer. The positive ions were accumulated in the hexapole ion guide for $0.2 \mathrm{~s}$ in Laboratory A and $0.05 \mathrm{~s}$ in Laboratory B and then transferred to the analyzer cylindrical ICR cell (Infinity Cell) by electrostatic ion optics. Data acquisition was performed using XMASS (Bruker, Billerica, MA), version 5.0.10 in Laboratory A and version 6.1 in Laboratory B. All acquisitions were recorded using $512 \mathrm{~K}$ data points, zero filled once and apodized using a sinm 


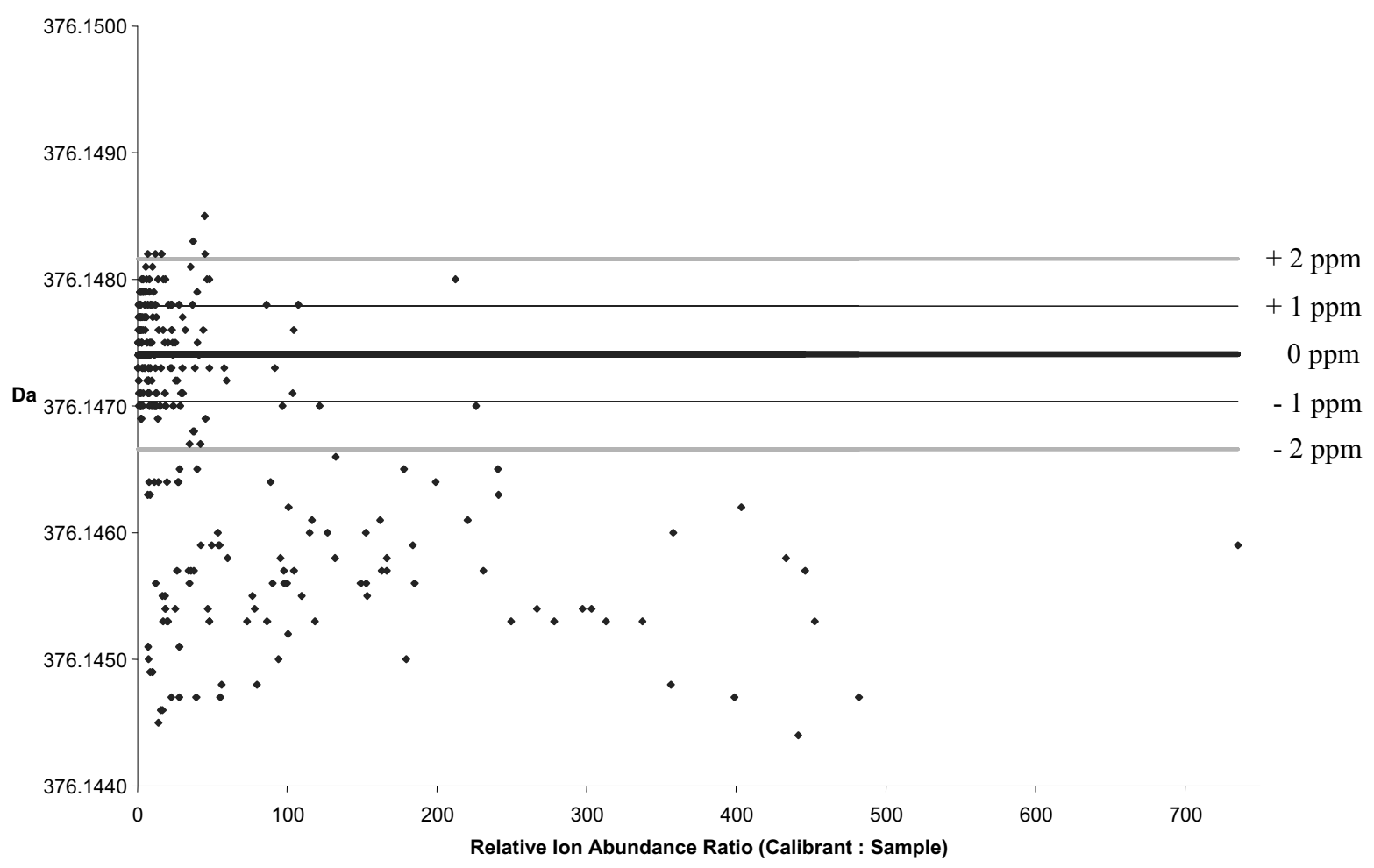

Figure 2. Graph illustrating the $\mathrm{m} / \mathrm{z}$ values for all exact mass measurements from Laboratory A plotted against the relative ion abundance ratio for calibrant:sample.

function. Four scans were summed to produce the final positive ion electrospray mass spectrum for each sample and calibrant. The scan range for each acquisition was $m / z 93.8-800$.

\section{Reagents, Standards, and Samples}

HPLC grade methanol (Fluka, Sigma-Aldrich, Gillingham, UK) was used as the solvent and formic acid (Avocado, Heysham, Lancashire, UK) used to aid the positive ion electrospray ionization process. The external calibration solution was prepared from diphenhydramine, oxybutynin chloride, terfenadine, reserpine and gramicidin S. The sample (haloperidol) and all calibrants were purchased from Sigma-Aldrich (Gillingham, UK).

Stock solutions of the individual calibrants were prepared at $1 \mathrm{mg} \mathrm{mL}^{-1}$ in methanol, and subsequent serial dilutions were performed using this solvent to produce calibration solutions that contained $0.1 \mu \mathrm{g}$ $\mathrm{mL}^{-1}, 1.0 \mu \mathrm{g} \mathrm{mL}^{-1}, 10 \mu \mathrm{g} \mathrm{mL} \mathrm{m}^{-1}$, and $100 \mu \mathrm{g} \mathrm{mL}^{-1}$ of each component with $0.1 \%$ formic acid added to the final solutions. The sample was prepared in an identical manner.

\section{External Calibration and Sample Analysis}

The calibration and sample solutions were infused using a Harvard syringe pump (Harvard Apparatus, Holliston, MA) at a constant flow rate of $1 \mu \mathrm{L} \mathrm{min}^{-1}$. A calibration at each of the concentration levels was performed using the Xmass software and these calibration files were used to externally calibrate the samples at each of the three concentrations.

\section{Results and Discussion}

Initially the data was plotted as measured mass $(\mathrm{m} / \mathrm{z})$ against relative ion abundance ratio of the calibration solution:sample solution. This is shown in Figure 2, for Laboratory A. Whilst this initial data plot is somewhat misleading in the format shown, as it does not show occurrences of identical measurements nor does it give a representation of the frequency of measurements at different error levels, it does show an overall trend towards lower observed mass measurement with increasing ratio of calibrant to sample; suggesting this is in agreement with the observations of Taylor and Amster [8]. Only by re-evaluating the data and calculating the frequency of occurrence of measurements at the different error levels can the performance of the individual machines be evaluated as shown in Figure 3a and $b$. From these pie-charts it can be seen that both systems behave in a very comparable manner up to the 2 ppm error range. It is noteworthy that the data for Laboratory A exhibits extended tailing of measurements with errors up to $8 \mathrm{ppm}$, whereas measurements for Laboratory B do not exceed $5 \mathrm{ppm}$. The reason for this difference in performance is not fully understood at this time but some of this may be due to the fact that 

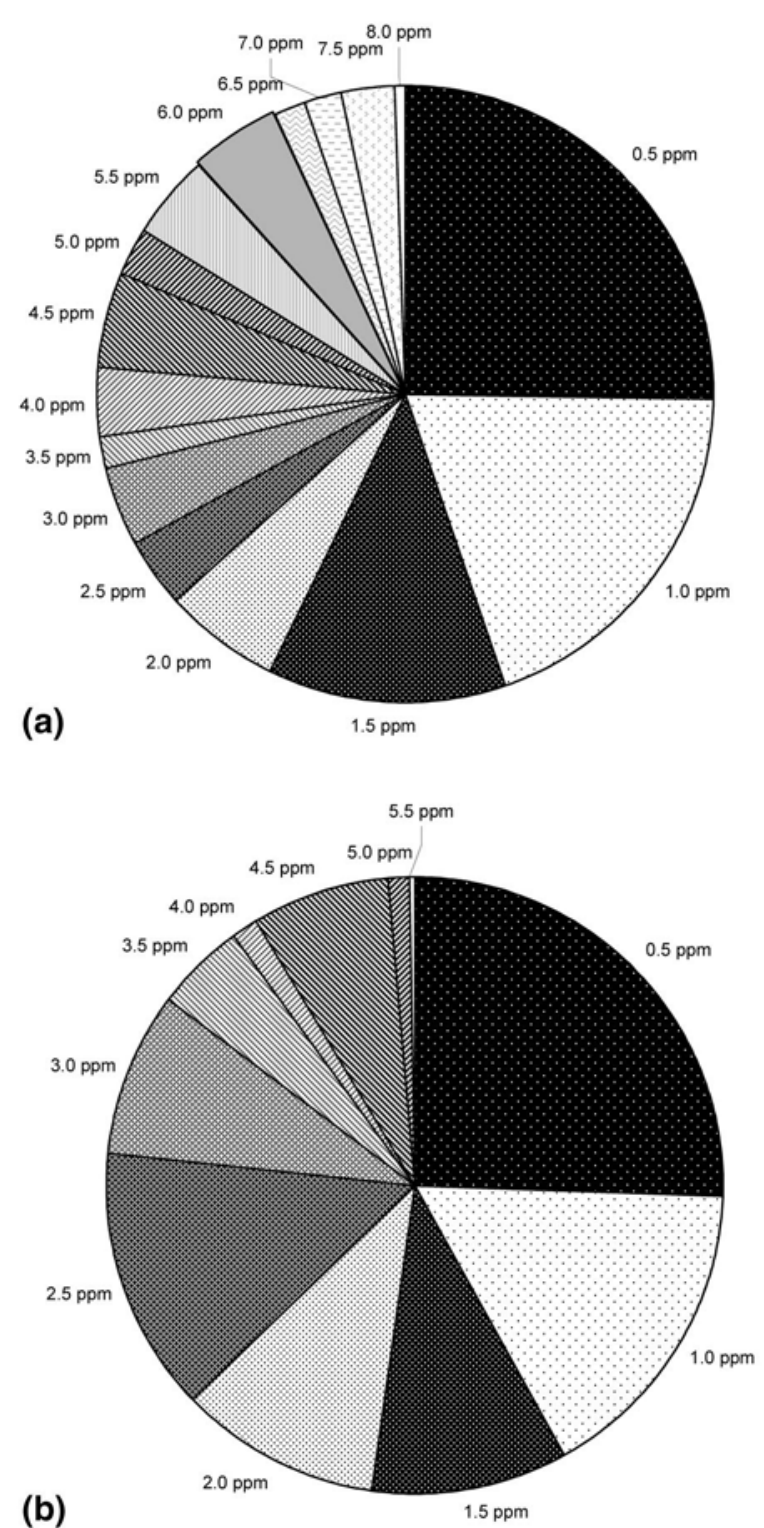

Figure 3. Pie-charts illustrating the percentages of measurements at the corresponding ppm errors for each laboratory, (a) and (b).

Instrument A showed a larger dynamic range for calibration, i.e., up to $100 \mu \mathrm{g} \mathrm{mL}^{-1}$ of each component, whereas peak splitting was observed for Instrument B at this calibration concentration.

Because of the large number of data points and complexity of the data, the dataset was subdivided to clarify the origin of the variability of the measurements. These data were then replotted for the individual sample concentration, i.e, Figure $4 \mathrm{a}$, b, and c shows this for Laboratory A at sample concentrations $0.1,1.0$ and 10 $\mu \mathrm{g} \mathrm{mL}^{-1}$. For ease of discussion, sample concentrations are referred to throughout, however it is the respective ion abundances that are relevant. From these figures a general trend was observed showing an increase in the error of mass measurement with an increase in the relative ion abundance ratio. This is in general agreement with expectations due to ion space charge effects [8].
To further understand the relationship between relative ion abundance and frequency of occurrence of mass measurement at specific accuracies, the original data sets were re-analyzed. The relative ion abundance ratios were grouped according to the following:

1. At each sample concentration level the relative ion abundance ratios were subdivided into arbitrary groups in order to assess the effect of increasing relative ion abundance ratio on the error of exact mass measurement.

2. For each arbitrary relative ion abundance group, e.g., RA $<5$, RA $<10$ etc., the number of occurrences of measurement under a specific ppm range was calculated.

3. The number of measurements at each specific ppm range were converted into a percentage to give final look-up tables (Table 2a, b and c), to be used for the assessment of measurement performance under the different experimental conditions.

As a result of this data sorting process, the tables allow generic statements concerning the frequency of occurrence of mass measurement at known relative ion abundance ratios to be made. Further, for Laboratory A, it can be seen that at low sample concentration, $0.1 \mu \mathrm{g}$ $\mathrm{mL}^{-1}$, all measurements under $3 \mathrm{ppm}$ correspond to a range in relative ion abundances, calibrant:sample, of up to 50:1, see shaded area of Table 2a. As the sample concentration increases, the relative ion abundance range corresponding to measurements of this accuracy, $100 \%$ under $3 \mathrm{ppm}$, decrease, i.e., relative ion abundance ratio up to $15: 1$ at $1.0 \mu \mathrm{g} \mathrm{mL}^{-1}$ and up to $5: 1$ for samples at $10 \mu \mathrm{g} \mathrm{mL}^{-1}$. Whilst the absolute relative ion abundance values differ for Laboratory B, the same overall trend was observed; e.g., for low sample concentration $\left(0.1 \mu \mathrm{g} \mathrm{mL} \mathrm{mL}^{-1}\right)$ at relative ion abundance ratios (calibrant:sample) of up to $40: 1,100 \%$ of measurements are below $4 \mathrm{ppm}$ and $96 \%$ are below $3 \mathrm{ppm}$, whereas at lower relative ion abundance ratios (calibrant:sample) up to $10: 1,100 \%$ of measurements are below 3 ppm and $73 \%$ are below 2 ppm.

One conclusion from these data is that, if the sample ion abundance is low, the accuracy and precision of the exact mass measurement is more tolerant of a wider range of calibration ion abundances, making this a valuable consideration for high throughput open access operation. A further consideration is that when the sample ion abundance is high, the calibration ion population must also be high to maintain the same level of confidence in the individual mass measurement, i.e., closer matching ion populations, hence the calibrant: sample ratio is low, see Table 2a. Consideration must be given at this stage as to whether $100 \%$ confidence limit is realistic as a working practice; shifting this paradigm to 98 or $95 \%$ alters the working range for expected measurements.

There are other aspects about the high ion abundance which must not be overlooked. High ion abun- 

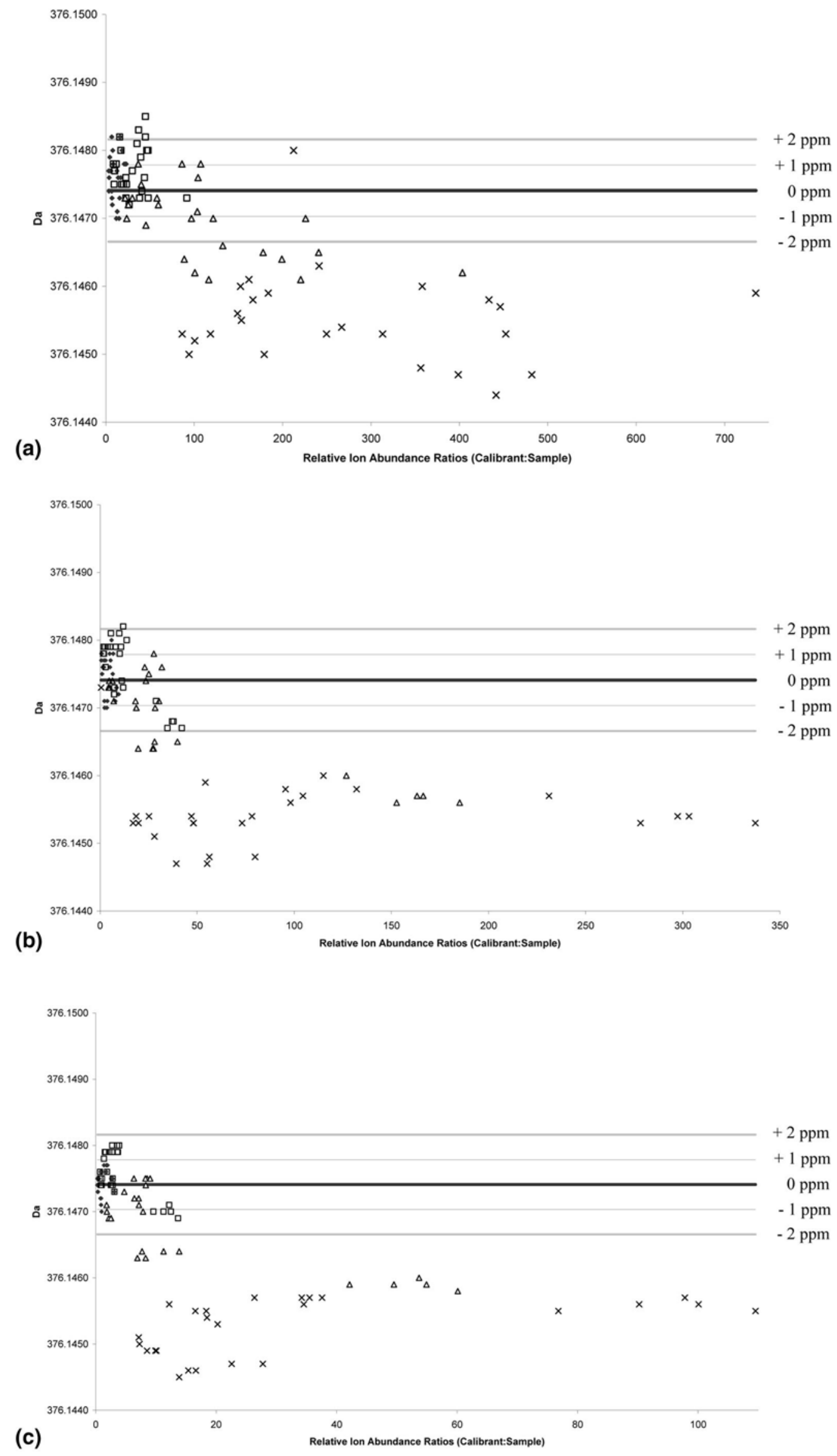

Figure 4. Graphs illustrating the $\mathrm{m} / \mathrm{z}$ values for exact mass measurements from Laboratory A plotted against the relative ion abundance ratio for calibrant:sample, where the sample is at a concentration of (a) $0.1 \mu \mathrm{g} \mathrm{mL}^{-1}$, (b) $1.0 \mu \mathrm{g} \mathrm{mL}^{-1}$, and (c) $10 \mu \mathrm{g} \mathrm{mL}^{-1}$. In each case the calibration concentration used is as follows: (filled diamond) $0.1 \mu \mathrm{g} \mathrm{mL}^{-1}$, (open square) $1.0 \mu \mathrm{g} \mathrm{mL}^{-1}$, (open triangle) $10 \mu \mathrm{g} \mathrm{mL}^{-1}$, and $(X) 100 \mu \mathrm{g} \mathrm{mL}^{-1}$. 
Table 2. The cumulative percentage of measurements below each nominal ppm error for Laboratory A, grouped according to relative ion abundance ratio for calibrant: sample where the sample concentration is (a) $0.1 \mu \mathrm{g} \mathrm{mL}^{-1}$ (b) $1.0 \mu \mathrm{g} \mathrm{mL}^{-1}$ and (c) $10 \mu \mathrm{g} \mathrm{mL}^{-1}$.

Table 2a.

\begin{tabular}{crrrrrrrr}
\hline \multirow{2}{*}{$\begin{array}{c}\text { Ratio Calibrant: } \\
\text { Sample }\end{array}$} & \multicolumn{8}{c}{ Cumulative error (ppm) } \\
\cline { 2 - 8 } & 1 & 2 & 3 & 4 & 5 & 6 & 7 & 8 \\
\hline \hline$<5$ & 80 & 100 & & & & & & \\
$<10$ & 80 & 93 & 100 & & & & & \\
$<15$ & 81 & 95 & 100 & & & & & \\
$<20$ & 69 & 90 & 100 & & & & & \\
$<25$ & 74 & 92 & 100 & & & & & \\
$<30$ & 75 & 93 & 100 & & & & & \\
$<35$ & 76 & 93 & 100 & & & & & \\
$<40$ & 72 & 91 & 100 & & & & & \\
$<50$ & 68 & 89 & 100 & & & & & \\
$<100$ & 66 & 86 & 97 & 97 & 97 & 98 & 100 & \\
$<150$ & 61 & 80 & 90 & 93 & 94 & 99 & 100 & \\
$<200$ & 55 & 72 & 84 & 90 & 93 & 98 & 100 & \\
$<300$ & 51 & 69 & 82 & 89 & 91 & 98 & 100 & \\
$>300$ & 45 & 61 & 73 & 81 & 86 & 94 & 97 & 100
\end{tabular}

Table $2 b$.

\begin{tabular}{|c|c|c|c|c|c|c|c|c|}
\hline \multirow{2}{*}{$\begin{array}{c}\text { Ratio Calibrant: } \\
\text { Sample }\end{array}$} & \multicolumn{8}{|c|}{ Cumulative error (ppm) } \\
\hline & 1 & 2 & 3 & 4 & 5 & 6 & 7 & 8 \\
\hline$<5$ & 76 & 100 & & & & & & \\
\hline$<10$ & 73 & 100 & & & & & & \\
\hline$<15$ & 70 & 98 & 100 & & & & & \\
\hline$<20$ & 63 & 91 & 95 & 95 & 95 & 100 & & \\
\hline$<25$ & 64 & 92 & 95 & 95 & 95 & 100 & & \\
\hline$<30$ & 60 & 85 & 90 & 93 & 93 & 98 & 100 & \\
\hline$<35$ & 61 & 86 & 90 & 93 & 93 & 99 & 100 & \\
\hline$<40$ & 57 & 84 & 89 & 92 & 97 & 99 & 100 & \\
\hline$<50$ & 54 & 81 & 86 & 89 & 89 & 97 & 99 & 100 \\
\hline$<100$ & 49 & 74 & 78 & 82 & 84 & 94 & 98 & 100 \\
\hline$<150$ & 47 & 70 & 75 & 80 & 86 & 96 & 99 & 100 \\
\hline$<500$ & 43 & 64 & 68 & 73 & 82 & 85 & 98 & 100 \\
\hline
\end{tabular}

Table 2c.

\begin{tabular}{ccccccccc}
\hline \multirow{2}{*}{$\begin{array}{c}\text { Ratio Calibrant: } \\
\text { Sample }\end{array}$} & \multicolumn{8}{c}{ Cumulative error (ppm) } \\
\cline { 2 - 9 } & 1 & 2 & 3 & 4 & 5 & 6 & 7 & 8 \\
\hline \hline$<5$ & 73 & 100 & & & & & & \\
$<10$ & 71 & 90 & 94 & 94 & 94 & 94 & 100 & \\
$<15$ & 60 & 84 & 91 & 91 & 92 & 92 & 99 & 100 \\
$<20$ & 56 & 79 & 85 & 85 & 87 & 91 & 98 & 100 \\
$<25$ & 55 & 77 & 83 & 83 & 84 & 86 & 95 & 100 \\
$<30$ & 53 & 76 & 81 & 81 & 84 & 88 & 94 & 100 \\
$<35$ & 52 & 74 & 80 & 80 & 84 & 89 & 94 & 100 \\
$<40$ & 51 & 72 & 78 & 78 & 84 & 89 & 94 & 100 \\
$<50$ & 50 & 71 & 76 & 78 & 85 & 89 & 95 & 100 \\
$<100$ & 47 & 66 & 71 & 76 & 85 & 90 & 95 & 100 \\
$<150$ & 46 & 65 & 70 & 74 & 84 & 90 & 95 & 100 \\
\hline
\end{tabular}

dance can cause peak splitting, but there is also an intrinsic observation that at high concentration for a single ion, where no peak splitting or loss of resolution is observed experimentally, the recorded mass error can be large. Simple sample dilution will improve the measurement accuracy.

Throughout these analyses the average errors, see Table $3 a, b$ and c, are always less than 3 ppm for all sample concentrations irrespective of the calibrant: sample ratio.

This data, whilst attractive, does not afford a confidence budget to any of the measurements; e.g., consider the final line in Table 3c (RA $<150)$; here the average mass error is $2.19 \pm 2.31 \mathrm{ppm}$, yet $30 \%$ of the measurements are over $3 \mathrm{ppm}$, see Table 2a. If the range of the elemental formula candidates list is determined by the average error, all measurements over $2.19 \mathrm{ppm}$ would be discounted, but by referring to the equivalent line in Table $2(\mathrm{RA}<150)$ it can be seen that all candidates up to $8 \mathrm{ppm}$ must be considered.

It is noteworthy that detailed examination of Table $3 a, b$ and $c$ reveals that the best average errors and standard deviations are observed where the ion populations of the calibration and sample are balanced, i.e., the first line in each section of the table for each sample concentration $(\mathrm{RA}<5)$. This is concurrent with present space charge discussions [8].

These initial hypotheses were subsequently challenged using a blind trial of 10 compounds, the concentration of these unknown to the analysts and no attempt made to control the ion abundance. Calibration solutions were prepared as previously and the ion abundances for these and the samples calculated. The samples were then grouped in accordance to the correlation of ion abundances with respect to the ion abundances of the three calibration solutions. The $\mathrm{m} / \mathrm{z}$ measured for each sample was calibrated with each of the calibration spectra. The mass errors for the data were then compared with that predicted from the preliminary protocol. From the latter, since all samples gave low ion abundance ratios, they therefore fit into the optimum working range of all three look-up tables (Table 2). Hence, the expected mass errors are predicted to be less than $3 \mathrm{ppm}$. This was the case for $95 \%$ of the measurements carried out. One measurement was consistently high for all calibrations. It may be that there are issues with the preparation of this sample that is causing the variance with the model. These issues could be attributed to the fact that the blind trial was carried out with samples from chemical synthesis and again this result challenges whether $100 \%$ confidence is a realistic experimental goal.

It is noteworthy that the two instruments exhibit markedly different capabilities with respect to ionization response. The range of relative ion abundance ratios (calibrant:sample) for Laboratory A being approximately 20 times that of Laboratory B, i.e., the latter exhibits peak splitting with the $100 \mu \mathrm{g} \mathrm{mL}^{-1}$ calibration solution. At this time, the reason for the differences in performance is not understood.

With the knowledge of absolute instrument performance determined, under strictly regulated conditions, this allows the confidence limit to be set for the range of 
Table 3. The average mass error (ppm) for each relative ion abundance ratio grouping for calibrant: sample for Laboratory A, for each sample concentration, i.e. (a) $0.1 \mu \mathrm{g} \mathrm{mL} \mathrm{mL}^{-1}$ (b) $1.0 \mu \mathrm{g}$ $\mathrm{mL}^{-1}$ and (c) $10 \mu \mathrm{g} \mathrm{mL}^{-1}$.

Table 3a.

\begin{tabular}{cc}
\hline Ratio Calibrant:Sample & $\begin{array}{c}\text { Average ppm error } \\
\text { (Standard deviation) }\end{array}$ \\
\hline \hline$<5$ & $0.68(0.46)$ \\
$<10$ & $0.79(0.54)$ \\
$<15$ & $0.80(0.49)$ \\
$<20$ & $0.90(0.59)$ \\
$<25$ & $0.84(0.55)$ \\
$<30$ & $0.83(0.55)$ \\
$<35$ & $0.81(0.54)$ \\
$<40$ & $0.87(0.59)$ \\
$<50$ & $0.92(0.66)$ \\
$<100$ & $1.09(1.12)$ \\
$<150$ & $1.33(1.40)$ \\
$<200$ & $1.59(1.60)$ \\
$<300$ & $1.72(1.66)$ \\
$>300$ & $2.13(2.03)$ \\
\hline
\end{tabular}

Table $3 b$.

\begin{tabular}{cc}
\hline Ratio Calibrant:Sample & $\begin{array}{c}\text { Average ppm error } \\
\text { (standard deviation) }\end{array}$ \\
\hline \hline$<5$ & $0.80(0.36)$ \\
$<10$ & $0.82(0.45)$ \\
$<15$ & $0.84(0.49)$ \\
$<20$ & $1.13(1.17)$ \\
$<25$ & $1.11(1.16)$ \\
$<30$ & $1.29(1.37)$ \\
$<35$ & $1.28(1.35)$ \\
$<40$ & $1.38(1.49)$ \\
$<50$ & $1.54(1.65)$ \\
$<100$ & $1.92(2.01)$ \\
$<150$ & $2.02(2.01)$ \\
$<500$ & $2.29(2.11)$ \\
\hline
\end{tabular}

Table 3c.

\begin{tabular}{cc}
\hline Ratio Calibrant:Sample & $\begin{array}{c}\text { Average ppm error } \\
\text { (standard deviation) }\end{array}$ \\
\hline \hline$<5$ & $0.64(0.50)$ \\
$<10$ & $1.06(1.51)$ \\
$<15$ & $1.32(1.79)$ \\
$<20$ & $1.61(2.10)$ \\
$<25$ & $1.72(2.20)$ \\
$<30$ & $1.82(2.28)$ \\
$<35$ & $1.88(2.29)$ \\
$<40$ & $1.94(2.30)$ \\
$<50$ & $1.99(2.29)$ \\
$<100$ & $2.14(2.30)$ \\
$<150$ & $2.19(2.31)$ \\
\hline
\end{tabular}

elemental formulas to be considered; i.e., all formulas under the stated ppm limit must be considered. Since the measurement capability is now known, the confidence limits for selection of elemental formulas can be proscribed based on sound experimental protocol and not arbitrary limits; e.g., in Table 1, where Example 2 shows a compound of measured nominal mass $\mathrm{m} / \mathrm{z} 500$, then all candidates must be considered under the $3 \mathrm{ppm}$ confidence limit determined by the experimental pro- tocol. In this case, there are 21 candidates for a simple species that could contain $\mathrm{C}, \mathrm{H}, \mathrm{N}, \mathrm{O}$, and $\mathrm{Na}$ and over 500 candidates if more elements are considered. Further rationalization of the possible candidates can then be carried out because of obeyance of the nitrogen rule, even electron species, simple valency compliance, isotope pattern, and other information known about the sample.

\section{Conclusions}

It is easy to be reassured and overconfident concerning the performance of an exact mass instrument since this is usually expressed in terms of the average mass measurement capability; this study has demonstrated that this can be misleading. It is the single mass measurement capability that is critical, and more so, having confidence in that single measurement that is important [9]. Therefore, for a species where the elemental formula is unknown, there is no recourse to judge the accuracy of the measurement other than by predetermination of the instrument capability in a manner similar to that described here. This is imperative for a high throughput, automated environment where operator intervention is desired to be minimal. It is the belief of the authors that all measurements should be validated regardless of whether you have any prior knowledge of the sample, and so the performance of any instrument must be defined and the results used to set the experimental error range of that instrument. This can then be used to determine the number of elemental formula candidates.

For this study, the relative ion abundance calculations are essential to determine the working ranges for specific sample ion abundances. Further, it has been shown that if the sample ion abundance is low, the ion abundance range for the calibration file does not need to be exactly or even closely matched. This observation is at variance with the commonly accepted view that matching of ion populations is critical to achieving the best accuracy for externally calibrated exact mass measurement. Whereas at high sample ion abundance closely matching ion populations are required, i.e., the calibrant:sample ratio is low. This being in agreement with the literature, where matching of ion populations is deemed critical to achieving the best accuracy for externally calibrated exact mass measurement

In this case, the data shows that the system is tolerant of a greater difference in ion populations, see Table 2, thus, suggesting that working at low ion abundance for samples would be best suited for automated data acquisition.

The reverse is true for high sample ion abundances; these require high calibration ion abundances and afford a narrow relative ion abundance working range to produce high accuracy measurements ( $<2 \mathrm{ppm}$, Table 2). At all times, the total ion abundance must be considered to avoid unreliable measurements and peak splitting.

Even though there are obvious differences between the performances of the two instruments, highlighted 
by the variation in dynamic range of ionization response, similar conclusions can be made about the optimum working relative abundance ranges and the confidence in the exact mass measurement recorded for each system.

The authors suggest that the experimental protocols such as those described in this paper should be applied to the validation of all exact mass measurements, i.e., the elemental formulas candidate(s) determined by known instrument performance for a single measurement. It is the confidence in the measurement carried out that is most important. While $100 \%$ confidence is the ideal scenario, a statistically sound experimental confidence limit that matches the needs of an individual laboratory is the overriding factor. For the first time generic statements concerning the frequency of mass measurement at known relative ion abundance ratios can be made.

\section{Acknowledgments}

The work described in this paper was supported under contract with the Department of Trade and Industry of the United Kingdom as part of the National Measurement System Valid Analytical Measurement (VAM) program.

\section{References}

1. Beynon, J. H. High Resolution Mass Spectrometry of Organic Materials. Adv. Mass. Spectrom. 1959, 328-354.

2. Guidelines to Authors. J. Org. Chem.

3. Marshall, A.G. Scaling MS Plateaus with High-Resolution FTICRMS. Anal. Chem. 2002, 74(9), 253A-259A.

4. Marshall, A. G.; Hendrickson, C. L.; Jackson, G. S. Fourier Transform Ion Cyclotron Resonance Mass Spectrometry; A Primer. Mass Spectrom. Rev. 1998, 17, 1-35.

5. Zhang, L.-K.; Rempel, D.; Pramanik, B. N.; Gross, M. L. Accurate Mass Measurements by Fourier Transform Mass Spectrometry. Mass Spectrom. Rev. 2005, 24, 286-309.

6. Bristow, A. W. T.; Webb, K. S. Intercomparison Study on Accurate Mass Measurement of Small Molecules in Mass Spectrometry. J. Am. Soc. Mass Spectrom. 2003, 14, 1086-1098.

7. Dykes, S.; Fancy, S.-A.; Perkins, G. L.; Pullen, F. S. The Automation of a Commercial Fourier Transform Mass Spectrometer to Provide a Quick and Robust Method for Determining Exact Mass for the Synthetic Chemist. Eur. J. Mass. Spectrom. 2003, 9, $80-73$.

8. Taylor, P. K.; Amster, I. J. Space Charge Effects on Mass Accuracy for Multiply-Charged Ions in FT-ICR. Int. J. Mass Spectrom. 2003, 222, 351-361.

9. Price, P. C.; Gale, P. J.; Loo, J. A.; Heller, D. N.; Richardson, S. D.; Duncan, M. W. ASMS Guidelines for Exact Mass Measurement and Elemental Composition-New Perspectives. Proceedings of the 50th Annual Conference on Mass Spectrometry and Allied Topics; Orlando, FL, 2002. 